Trabajos Originales

\title{
Tumor de colisión de cérvix uterino: presentación de 17 casos
}

\author{
Ernesto García A. ${ }^{1}$, Carlos Alberto García R. ${ }^{2}$, Diana Sandoval M. ${ }^{1}$ \\ ${ }^{1}$ Departamento de Patología, Facultad de Salud, Universidad Industrial de Santander; ${ }^{2}$ Departamento de Patología, \\ Facultad de Salud, Universidad Autónoma de Bucaramanga. Colombia.
}

\section{RESUMEN}

El tumor de colisión es una variante poco frecuente de tumores del cérvix uterino, originados de las células de reserva de la unión escamocelular y que debe ser diferenciado de los carcinomas adenoescamosos. El objetivo del presente artículo es determinar las características morfológicas de 17 casos de tumor de colisión de cérvix uterino. La edad promedio de presentación fue 48,7 años (rango: $34-87$ años). Los 4 casos con metástasis ganglionares correspondieron al componente de adenocarcinoma.

\section{PALABRAS CLAVE: Cérvix, tumor de colisión, tumor adenoescamoso, tumor mixto}

\section{SUMMARY}

Colison tumor is an uncommon variant of cervical tumors originating from the reserve cells of squamous cell union and must be differentiated from adenosquamous carcinomas. The aim of this paper is to determine the morphological characteristics of 17 cases of collision tumor of uterine cervix, in which it was found that the average age at diagnosis was 48.7 years (range: $34-87$ years). In 5 cases lymph node metastases were of the adenocarcinoma component.

KEY WORDS: Cervix, collision tumors, adenosquamous tumor, mixed tumor

\section{INTRODUCCIÓN}

El cáncer de cuello uterino es la segunda neoplasia maligna más frecuente en las mujeres residentes del Área Metropolitana de Bucaramanga (Santander/Colombia), correspondiendo al 14,1\% de los nuevos casos de cáncer diagnosticados en mujeres, con un promedio anual de 95 casos (2 casos nuevos por semana) (1), se considera que el $80 \%$ de estos son alguna variante de carcinoma escamocelular, el $15 \%$ adenocarcinomas o sus subtipos y el $5 \%$ restante otros tumores (2-11).

Aunque esta claramente establecido que los carcinomas escamosos pueden tener diferenciación adenoide o por el contrario los adenocarcinomas presentar diferenciación escamoide (5$7,9,10,12$ ), los tumores que contienen mezclas histológicas de células escamosas y glandulares malignas se conocen con el nombre de carcinomas adenoescamosos (3,5-9,11-13) y deben ser diferenciados del llamado tumor de colisión del cérvix (TCC), en el cual los componentes de adenocarcinoma y carcinoma escamocelular son claramente distinguibles y se encuentran separados entre si por una banda estrecha de estroma (o su lámina basal) $(4,7,9,10,14)$. Se ha demostrado que 
las células basales de la unión escamocolumnar funcionan como células de reserva y que pueden dar origen, durante eventos reparativos, a epitelio escamoso o columnar $(3,5-7,8-11,15)$, y a su vez, ante condiciones adversas del medio, como eventos cancerígenos específicos como la infección por VPH $(8,15)$, estimulación hormonal del embarazo (14), dar origen a sus contrapartes tumorales, carcinoma escamocelular y adenoacarcinomas (3,511), esto se correlaciona con la alta incidencia de la coexistencia de alteraciones de tipo displásico en eventos invasores de uno u otro tipo (8-15). La importancia de este tipo de tumor está relacionado con un peor pronóstico para las pacientes en relación directa al mayor grado tumoral de las lesiones invasoras $(7,13,15,16)$.

No existen revisiones recientes acerca de las características epidemiológicas de los TCC. El objetivo de esta comunicación es determinar las características morfológicas de 17 casos de este tipo de lesiones.

\section{PACIENTES Y MÉTODO}

Se revisó la base de datos de 2 Laboratorios de Patología de la Ciudad de Bucaramanga, Colombia, desde el año 2002 a 2009. Se detectaron 17 casos de TCC, que cumplía con los criterios histológicos de tener los dos componentes tumorales separados por estroma y que en ningún momento existiera transición entre ellos $(4,7-10,14)$. Las muestras de estas pacientes y sus historias clínicas fueron analizadas nuevamente por un grupo de tres patólogos. Las descripciones se basaron en el examen microscópico de las secciones histológicas disponibles (bloques de tejido embebidos en parafina), en los 17 casos analizados hubo bloqueo seriado de todo el cérvix uterino por lo que se evaluó la muestra tumoral completa. El prerrequisito del examen fue determinar los puntos de origen y las relaciones definitivas de los dos elementos que intervienen.

\section{RESULTADOS}

Se encontraron 17 casos de TCC (Tabla I), que cumplía con los criterios histológicos de inclusión al estudio (Figura 1a y 1b). En 4 casos se evidenciaron metástasis ganglionares con un total de 21 de 59 ganglios linfáticos comprometidos (Tabla II), dichas metástasis correspondieron en su totalidad al componente de adenocarcinoma de todos los casos (Figura 2).

Con relación a las variantes histológicas se encontró con respecto al componente de adenocarcinoma que el $58,8 \%$ de las lesiones fueron invasoras (10 casos) y los 7 casos restantes correspondieron a adenocarcinomas in situ $(41,2 \%)$. De las lesiones invasoras el subtipo más frecuente fue el de adenocarcinoma mucinoso de tipo endocervical con 6 casos (35,3\%) seguido del adenocarcinoma endometrioide con 3 casos (17,6\%), reportando 1 caso de adenocarcinoma adenoide quístico (5,9\%) (Figura 1a).

Por su parte, el componente escamoso en el $41,7 \%$ de los casos ( 7 casos) se trató de carcinoma in situ, 5 casos $(29,4 \%)$ fueron tumores microinvasores y el mismo porcentaje $(29,4 \%)$ es decir 5 casos fueron invasores, de los cuales $3(17,6 \%)$ fueron moderadamente diferenciados y $11,8 \%$ (2 casos) bien diferenciados.

Los porcentajes de las mezclas encontradas fueron: 1. Ca infiltrante + Ca infiltrante: 4 casos (23,5\%); 2. Ca infiltrante + Ca microinvasor: 3 casos $(17,6 \%)$; 4. Ca infiltrante + $\mathrm{Ca}$ in situ: 4 casos $(23,5 \%) ; 5$. Ca microinvasor + Ca in situ: 2 casos $(11,8 \%) ; 5$. Ca in situ + Ca in situ: 4 casos (23,5\%) (Figura 1b).

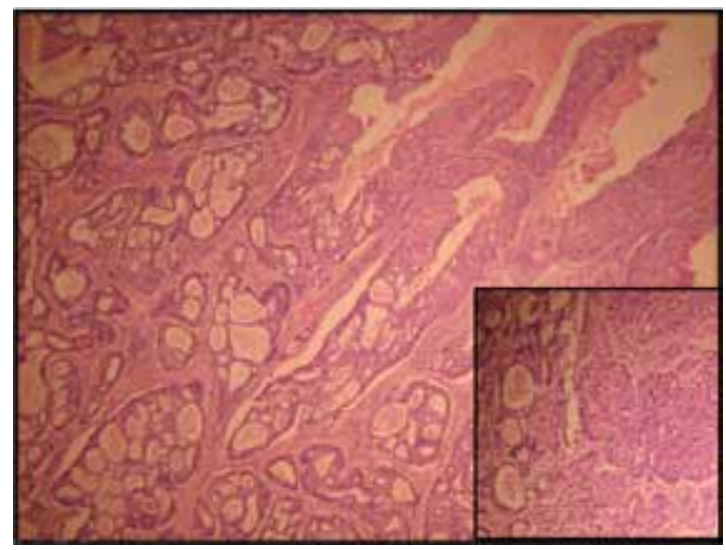

Figura 1a. Caso 12. Adenocarcinoma de endocérvix de tipo adenoide quístico infiltrante + $\mathrm{Ca}$ escamoso moderadamente diferenciado infiltrante.

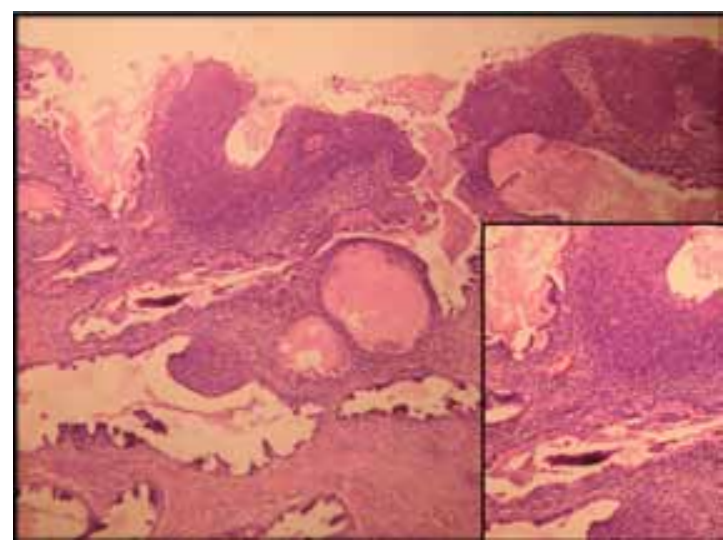

Figura 1b. Caso 16. Adenocarcinoma endocervical in situ + Ca escamoso in situ con extensión glandular. 
Tabla I

CARACTERIZACION DE LOS 17 CASOS DE TUMOR DE COLISIÓN DEL CÉRVIX UTERINO

\begin{tabular}{|c|c|c|c|}
\hline Caso & Edad & Estadio & Diagnóstico \\
\hline 1 & 68 & IIIB & $\begin{array}{l}\text { Adenocarcinoma mucinoso de tipo endocervical infiltrante }+\mathrm{Ca} \\
\text { escamoso moderadamente diferenciado infiltrante }\end{array}$ \\
\hline 2 & 52 & IB & $\begin{array}{l}\text { Adenocarcinoma in situ }+ \text { Ca escamoso bien diferenciado infil- } \\
\text { trante }\end{array}$ \\
\hline 3 & 48 & IB & $\begin{array}{l}\text { Adenocarcinoma endometriode de endocérvix infiltrante }+\mathrm{Ca} \\
\text { escamoso in situ con extensión glandular }\end{array}$ \\
\hline 4 & 40 & 0 & $\begin{array}{l}\text { Adenocarcinoma in situ + Ca escamoso in situ con extensión } \\
\text { glandular }\end{array}$ \\
\hline 5 & 87 & IIA & $\begin{array}{l}\text { Adenocarcinoma mucinoso de tipo endocervical infiltrante+ } \mathrm{Ca} \\
\text { escamoso bien diferenciado infiltrante }\end{array}$ \\
\hline 6 & 46 & IB & $\begin{array}{l}\text { Adenocarcinoma mucinoso de tipo endocervical infiltrante }+\mathrm{Ca} \\
\text { escamocelular microinvasor }(3 \mathrm{~mm})\end{array}$ \\
\hline 7 & 39 & 0 & Adenocarcinoma in situ + Ca escamocelular in situ \\
\hline 8 & 35 & IB & $\begin{array}{l}\text { Adenocarcinoma mucinoso de tipo endocervical infiltrante }+\mathrm{Ca} \\
\text { escamocelular in situ }\end{array}$ \\
\hline 9 & 46 & IA2 & Adenocarcinoma in situ + Ca escamoso microinvasor $(3 \mathrm{~mm})$ \\
\hline 10 & 44 & IIIB & $\begin{array}{l}\text { Adenocarcinoma mucinoso de tipo endocervical infiltrante }+\mathrm{Ca} \\
\text { escamoso moderadamente diferenciado infiltrante }\end{array}$ \\
\hline 11 & 40 & 0 & $\begin{array}{l}\text { Adenocarcinoma endocervical in situ + Carcinoma escamocelu- } \\
\text { lar in situ con extension glandular }\end{array}$ \\
\hline 12 & 34 & IIIB & $\begin{array}{l}\text { Adenocarcinoma de endocérvix de tipo adenoide quistico infil- } \\
\text { trante + Ca escamoso moderadamente diferenciado infiltrante }\end{array}$ \\
\hline 13 & 52 & IB & $\begin{array}{l}\text { Adenocarcinoma endometrioide de endocérvix infiltrante }+\mathrm{Ca} \\
\text { escamocelular microinvasor }(2 \mathrm{~mm})\end{array}$ \\
\hline 14 & 64 & IIIB & $\begin{array}{l}\text { Adenocarcinoma endometrioide de endocérvix infiltrante }+\mathrm{Ca} \\
\text { escamocelular microinvasor }(2 \mathrm{~mm})\end{array}$ \\
\hline 15 & 47 & IB & $\begin{array}{l}\text { Adenocarcinoma mucinoso de tipo endocervical infiltrante }+\mathrm{Ca} \\
\text { escamocelular in situ }\end{array}$ \\
\hline 16 & 50 & 0 & $\begin{array}{l}\text { Adenocarcinoma endocervical in situ + Ca escamoso in situ con } \\
\text { extensión glandular }\end{array}$ \\
\hline 17 & 37 & $\mathrm{IA} 2$ & $\begin{array}{l}\text { Adenocarcinoma endocervical in situ + Ca escamoso microin- } \\
\text { vasor }(2 \mathrm{~mm})\end{array}$ \\
\hline
\end{tabular}

\section{DISCUSIÓN}

El tumor de colisión del cérvix fue por primera vez descrito en 1964 por Dougherty y Cotton (9) quienes hicieron una revisión de casos del Charity Hospital in New Orleans, LA., desde 1937 a 1963, y del Baton Rouge Pelvic Tumor Registry desde 1951 a 1962, encontrando 49 casos de tumores del cérvix uterino con componente tanto de carcinoma escamocelular como de adenocarcinoma, que fueron divididos en tres subvariantes de acuerdo a las características histológicas: 1. Carcinoma escamocelular y adenocarcinoma sincrónico del cérvix uterino; 2. Carcinomas adenoescamosos de cérvix uterino y 3. Carcinoma de colisión del cérvix uterino al que llamaron "tumor mixto", el cual se caracterizaba por encontrarse dichos componentes en el mismo campo o glándula endocervical y en los cuales, adicionalmente, no se veía transición entre ellos y había una banda de estroma o lámina basal que los separaba.

Posteriormente Choo y Nailor (14), denominaron tumor de colisión a este tipo de lesiones y consideraron que se originaban de las células de reserva de la zona de transformación, lo que fue corroborado por otros autores (5-11). En el 2000, Kersemaekers 


\section{Tabla II \\ CASOS TUMOR DE COLISIÓN DEL CÉRVIX UTERINO CON METÁSTASIS A GANGLIOS REGIONALES}

\begin{tabular}{lll}
\hline Caso & Metástasis & Tratamiento \\
\hline 1 & $5 / 25$ ganglios linfáticos regionales & Histerectomía + SOB \\
10 & $4 / 10$ ganglios linfáticos obturadores derechos & Histerectomía abdominal ampliada + \\
& y parametrio derecho & linfadenectomía pélvica \\
12 & 2 ganglios linfáticos obturadores derechos, & Histerectomía abdominal ampliada + \\
& 2 ganglios linfáticos obturadores izquierdos, 2 ganglios de & SOB + linfadenectomía pélvica \\
& iliaca primitiva izquierda & \\
14 & $\begin{array}{l}1 / 9 \text { ganglios linfáticos iliacos externos derechos-iliaca } \\
\text { primitiva, } \\
\end{array}$ & Histerectomía abdominal ampliada + \\
& metrio derecho y $1 / 4$ ganglios de parametrio izquierdo & SOB + linfadenectomía pélvica
\end{tabular}

SOB: salpingoooforectomía bilateral.

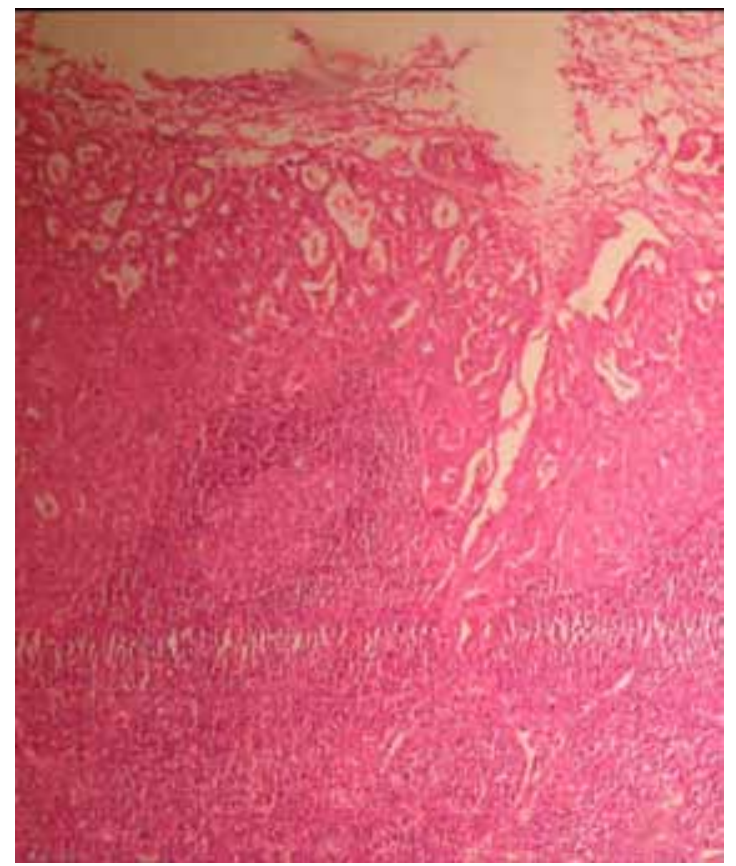

Figura 2. Caso 14. Ganglio linfático con compromiso por adenocarcinoma endometrioide de endocérvix.

y cols (4) demostraron por epigenética que los componentes de adenocarcinoma y de carcinoma de células escamosas de cada tumor tienen una célula precursora única, la cual se encuentra ubicada en la zona de la unión escamocolumnar, con el subsecuente desarrollo monoclonal en dos vías diferentes.
Reportamos 17 casos de tumor de colisión del cérvix los cuales se presentaron en un rango de edad entre los 34 y 87 años, con un edad promedio de presentación de 48,7 años, que coincide con lo encontrado por Dougherty y Cotton en 1964 (9), quienes observaron una mediana de presentación de 52 años con un rango que variaba entre los 28 y 80 años en los llamados entonces tumores mixtos, edades de presentación promedio inferiores a las presentadas en las pacientes con adenocarcinomas puros, esto adquiere importancia si se tiene en cuenta que el componente de adenocarcinoma fue el encontrado en las metástasis ganglionares, y que se ha demostrado que estos tumores presentan compromiso ganglionar pélvico dos veces más frecuente que sus contrapartes puras, lo que influye en un pobre y peor pronóstico en estas pacientes $(7,13,16,17)$.

\section{BIBLIOGRAFÍA}

1. Uribe JC, Meza EE. Incidencia de cáncer en el Área Metropolitana de Bucaramanga, 2000-2004. MedUNAB 2007;10:147-72.

2. Smith HO, Tiffany MF, Qualls CR, Key CR. The rising incidence of adenocarcinoma relative to squamous cell carcinoma of the cervix in the United States - a 24 year population - based study. Gynecol Oncol 2000;78:97-105.

3. Fletcher C. Diagnostic Histopathology of Tumors. 3ra ed. Elsevier. 2007, p. 702-15.

4. Kersemaekers AM, van de Vijver MJ, Fleuren GJ. Comparison of the genetic alterations in two epithelial collision tumors of the uterine cervix. A report of two cases. Int J Gynecol Pathol 2000;19:225-30.

5. Rosai J. Rosai and Ackerman's Surgical Pathology. Ninth edition. Ed: Mosby; 2004. pp 1534-51. 
6. Well M, Östör AG. Pathology and Genetics. Tumors of the breast and female genital organs. IARC. 2003. pp 262-279.

7. Kurman RJ. Blaustein's Pathology of the Female Genital Tract. 6th ed. Springer. 2011. Cap 6. Witkiewicz AK, Wright TC. Carcinoma and other tumors of the cervix. pp 254-303

8. Tase T, Okagaki T. Human papillomavirus types and localization in adenocarcinoma and adenosquamosous carcinoma of the uterine cervix: a study by in situ DNA hybridation. Cancer Research 1988;48:993-8.

9. Dougherty $\mathrm{CM}$, Cotton N. Mixed squamous-cell and adenocarcinoma of the cervix. Combined, adenosquamous, and mucoepidermoid types. Cancer 1964;17:1132-43.

10. Lauchlan SC, Penner DW. Simultaneous adenocarcinoma in situ and epidermoid carcinoma in situ. Report of two cases. Cancer 1967;20:2250-4.

11. Christopherson WM, Nealon N, Gray LA. Noninvasive precursor lesions of adenocarcinoma and mixed adenosquamous carcinoma of the cervix uteri. Cancer 1979;44:975-83.

12. van Aspert-van Erp AJ, Smedts FM, Vooijs GP. Seve- re cervical glandular cell lesions with coexisting squamous cell lesions. Cancer 2004;102:218-27.

13. Farley JH, Hickey KW, Carlson JW, Rose GS, Kost ER, Harrison TA. Adenosquamous histology predicts a poor outcome for patients with advanced-stage, but not early-stage, cervical carcinoma. Cancer 2003;97:2196-202.

14. Choo YC, Naylor B. Coexistent squamous cell carcinoma and adenocarcinoma of the uterine cervix. Gynecol Oncol 1984;17:168-74.

15. Ueda Y, Miyatake T, Okazawa M, Kimura T, Miyake T, Fujiwara K, Yoshino K, Nakashima R, Fujita M, Enomoto $\mathrm{T}$. Clonality and VPH infection analysis of concurrent glandular and squamous lesions of the uterine cérvix. Am J Clin Pathol 2008;130: 389-400.

16. Vesterinen E, Forss M, Nieminen U. Increase of the cervical adenocarcinoma: a report of 520 cases of cervical carcinoma including 112 tumors with glandular elements. Gynecol Oncol 1989;33:49-53.

17. Saigo PE, Cain JM, Kim WS, Gaynor JJ, Johnson K, Lewis JL Jr. Prognostic factors in adenocarcinoma of the uterine cervix. Cancer 1986;57:1584-93. 